\title{
Agent-Based Modeling of Physical Activity Impact on Health Benefit and Risk
}

\author{
Zaiyong Tang \\ Dept. of Marketing and Decision Sciences \\ Salem State University \\ USA \\ Fuping Zhu \\ Dept. of Industrial Engineering \\ Southwest University of Science and Technology \\ China
}

\begin{abstract}
We propose using agent-based modeling to study the impact of physical activity on health benefit and risk. Previous researches have focused on aggregated analysis at the population or group level, making recommendations that are useful, but may not be appropriate for individuals with substantially different health/risk profiles. The proposed model explores individualized recommendations of physical activity levels for risk adjusted health benefits while also allows population level analysis ofsocietal health benefits and system dynamics.
\end{abstract}

Keywords: Physical activity, health benefit, benefit/risk profile, agent-based modeling

\section{Introduction}

Numerous studies have shown that sports and physical activities lead to a wide range of benefits in social, psychological, and physical health (Kesaniemi et al., 2001). Although our understanding of health benefits and risks resulting from physical activities has evolved over the years, it has long been accepted that regular physical activity contributes to improved quality of life, lowering risk of various diseases, and reducing mortality rate (Melzer et al. 2004; Warburton et al., 2016). Supported by rigorous scientific research findings, government agencies and health professional organizations have issues recommendations since the 1970s. The American College of Sports Medicine and the Centers for Disease Control and Prevention published national guidelines on physical activity and public health in 1995. The recommendations were updated in 2007 (Haskell et al., 2007). The US Department of Health and Human Services issued Physical Activity Guidelines for Americans (U.S. DoHHS, 2008).The ABC of Physical Activity for Health: A consensus statement from the British Association of Sport and Exercise Sciences (O'Donovan et al., 2010) provided separate recommendations for all healthy adults (A), beginners (B), and conditioned individuals (C).

The US recommendation stipulates that, using inactivity as the baseline, low level $(<150$ minutes/week) moderate intensity physical activity provides some health benefits while moderate level (150-300 minutes/week) of moderate intensity activity provides substantial health benefits. Activity duration can be offset by intensity. For the same amount of health benefit, one minute of vigorous intensity activity is roughly equivalent to two minutes of moderate intensity activity (U.S. DoHHS, 2008). The British recommendation for all healthy adults is similar to the American recommendation: At least 150 minutes/week of moderate intensity aerobic activity, or at least 75 minutes/week of vigorous intensity aerobic activity. For beginners, it is recommended that they start at low level physical activity and work progressively towards reaching the activity levels recommended for "all healthy adults". Conditioned individuals are those who have reached the physical activity levels recommended for "all healthy adults" for at least six months. It is recommended that they engage in 300 minutes/week or more of moderate intensity aerobic activity, or 150 minutes/week or more of vigorous intensity aerobic activity to achieve further health benefits. No doubt that physical activity is beneficial. However, the relationship between physical activity and potential health consequences is very complex as it involves many factors such as type of activity, intensity, duration, age, gender, health status, and other personal traits. There is no consensus on the optimum or the minimum level of physical activity that is needed to achieve health benefits (Warburton et. al., 2016). 
The professional/government recommendations are useful guidelines but may not be appropriate for individuals with substantially different health/risk profiles. For example, an inactive person may consider the recommended level of activity intensity unattainable, while an avid athlete may feel the recommended level totally inadequate. We need to explore the possibility of individualized recommendation based on a person's health/risk profile.

In the next section, we review the literature on physical activity induced benefits and risks. We focus on the doseresponse relationship between physical activity and health benefits. Then, we cover briefly agent-based modeling and present our model for studying individualized health benefit/risk analysis. The model simulation results are presented next, followed by a discussion of contributions and limitations, and conclusions.

\section{Physical Activity Benefits and Risks}

Physical activity refers to any bodily movement produced by skeletal muscles that results in energy expenditure beyond resting expenditure (Melzer et al., 2004). The health benefits of physical activity have been recognized more than 2000 years ago (Warburton et. al., 2016). Plato stated that "Lack of activity destroys the good condition of every human being, while movement and methodical physical exercise save it and preserve it." (Conservapedia)

Typical benefits of physical activity include reduced risk of coronary heart disease, obesity, type 2 diabetes, osteopenia, sarcopenia, psychological disorder, etc. (Melzer et al., 2004). It is generally accepted that a higher level of physical activity leads to a higher level of health benefits. However, high intensity activityalso results in higher chance of injury and other risks (Kesaniemi et al., 2001). Extended physical activity may lead to musculoskeletal injury, oxidative stress, sudden cardiac death, dehydration, and heat stroke (Melzer et al., 2004). A commonly deployed measure for intensity of physical activity is metabolic equivalent task (MET). The energy expenditure at rest (sitting position) is defined as 1 MET. Table 1 lists a sample of physical activities and their associated number of METs. As shown in the table, moderate physical activity has a range of 3-6 METs. Less than 3 METs is considered light activity while more than 6 METs is considered vigorous activity (Ainsworth et al. 1993).

Table1. Sample Physical Activities and Energy Expenditure Measured in METs

\begin{tabular}{|c|c|c|}
\hline $\begin{array}{l}\text { Light Physical Activity } \\
\text { (<3 METs) }\end{array}$ & $\begin{array}{l}\text { Moderate Physical Activity (3- } \\
6 \text { METs) }\end{array}$ & $\begin{array}{l}\text { Vigorous Physical Activity } \\
\text { (> } 6 \text { METs) }\end{array}$ \\
\hline Activity $\quad$ METs & Activity $\quad$ METs & Activity $\quad$ METs \\
\hline Resting(sitting) 1.0 & Frisbee playing 3.0 & Jogging \\
\hline Standing $\quad 1.2$ & Walking(fast) 3.5 & Running $\quad 8.0$ \\
\hline Walking(slow) 2.0 & Table tennis 4.0 & Swimming(butterfly) 11.0 \\
\hline Playing piano 2.5 & Dancing(fast) 5.5 & Boxing(in ring) 12.0 \\
\hline
\end{tabular}

(Source: Ainsworth et al. 1993)

The health benefits from regular physical activity result from the acute and chronic physiologic changes. Regular physical activity benefits not only healthy individuals, it also brings therapeutic effect to people suffering various diseases. Kujala (2009) presented evidence-based pathways on how physical activity or exercise therapy delays progression of diseases and occurrence of disability and deaths. Figure 1 is a map recreated from Kujala (2009) showing the effect of physical activity on various factors that are linked to disability and/or death. The map was created by using Kumu, a data visualization platform (https://kumu.io).Kumuoffersa versatile set of tools for turning complex data into interactive maps, allowing data visualization with different perspectives. For example, Figure 2 is a subset of the map in Figure 1, showing only the direct impact of physical activity on disease-specific mechanisms. 


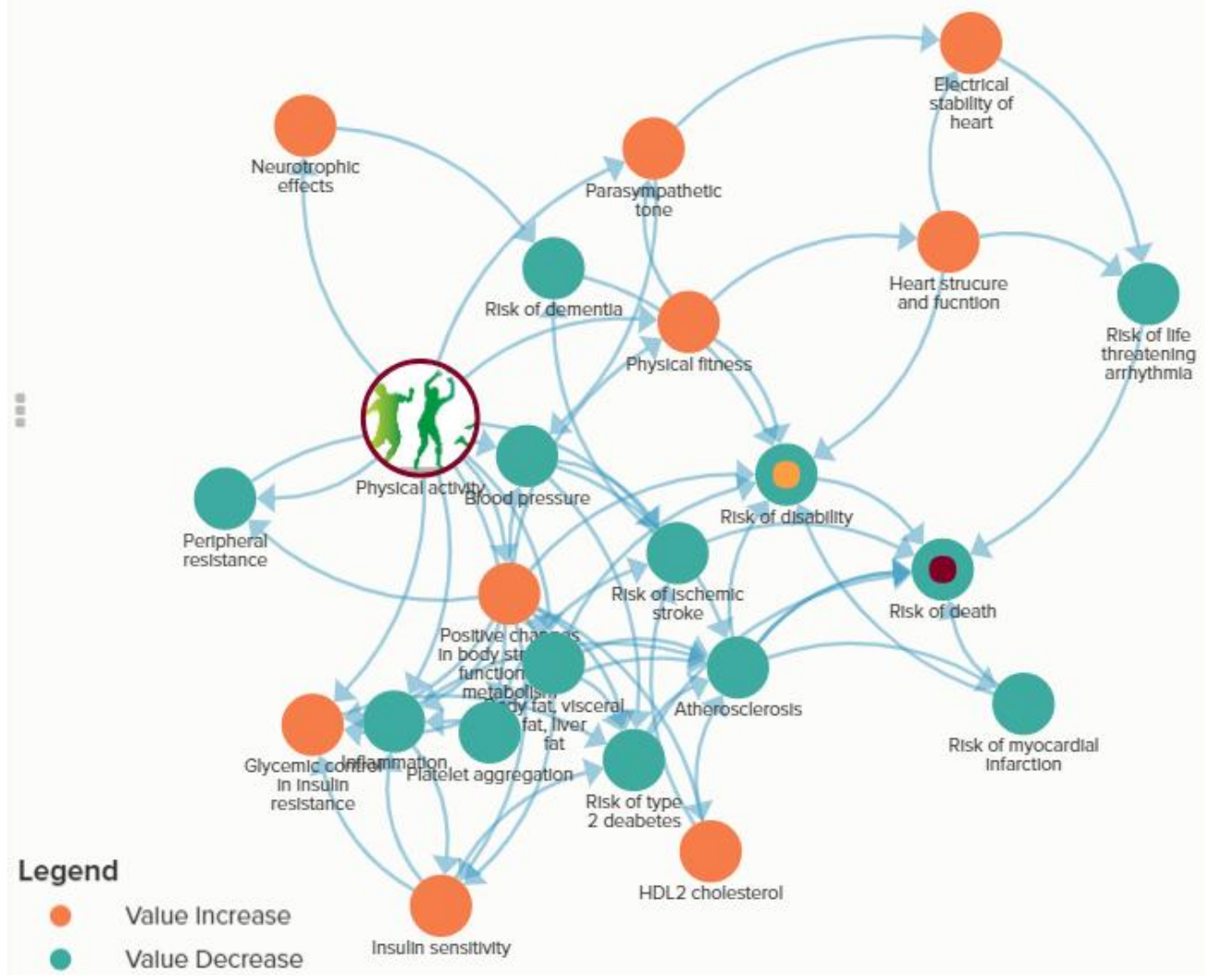

Figure 1. Impact of Physical Activity on Health Risk Factors

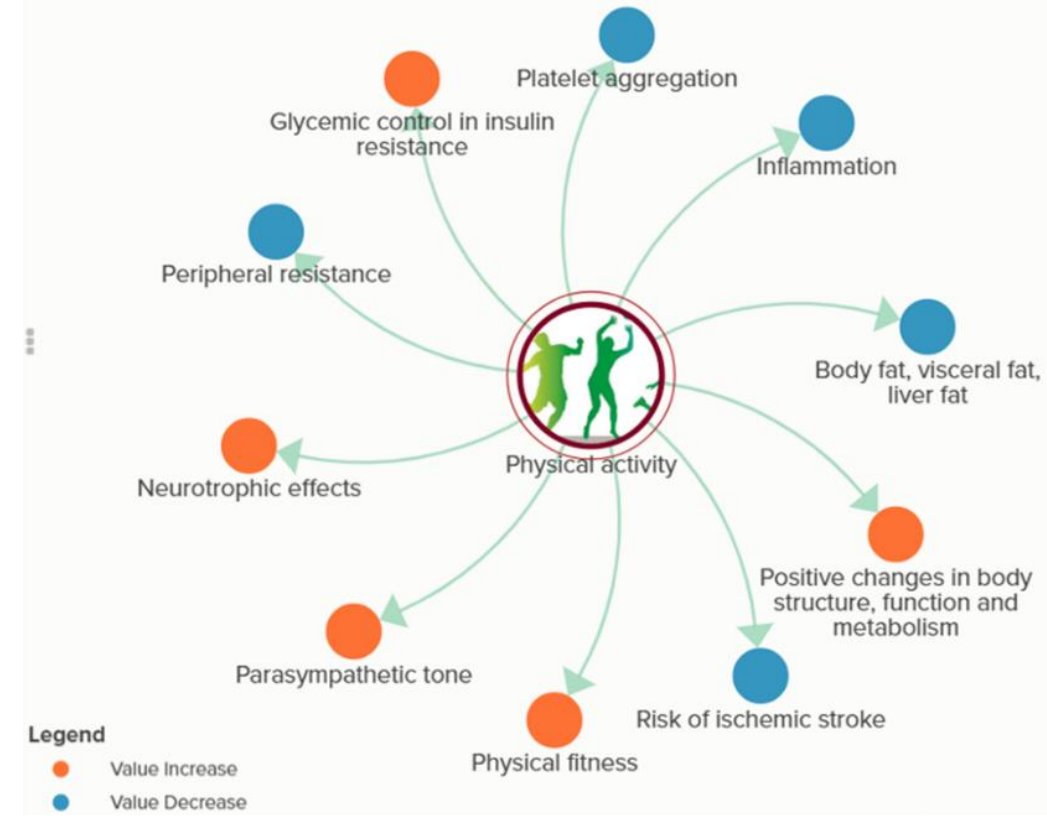

Figure2. Direct Impact of Physical Activity on Disease-Specific Mechanisms

Most research literature on physical activity has focused on the type and extend of health benefits. Nevertheless, researchers have acknowledged the risk-benefit paradox of exercise (Warburton et al. 2016). Gledhill (2003) identified different temporal relationship between physical activity and various determinants of health status, such as blood pressure, triglycerides, high-density lipoprotein, and body composition. 
Kesaniemi et al. (2001) suggested that individuals have different physical activity benefit profiles as shown in Figure 3, as well as different risk profiles. The recommended intensity of physical activity should consider the net health benefit, which is the difference between benefit and risk.

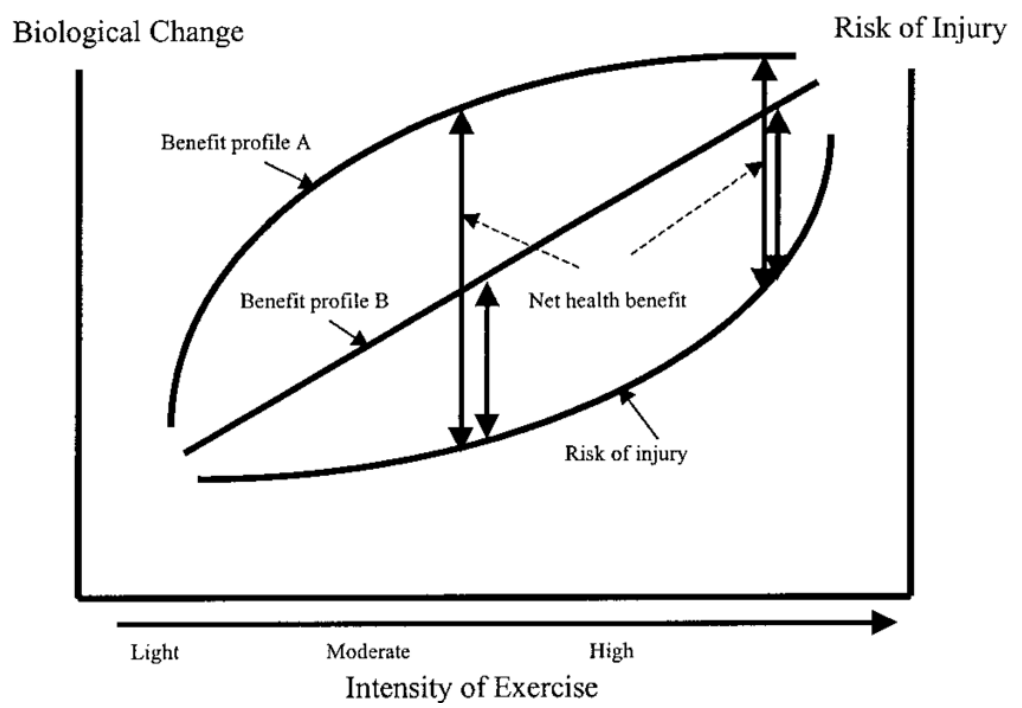

Figure 3. The Relationship of Exercise Intensity to Health Benefit and Risk (Source: Kesaniemi et al. 2001) Clearly, the largest net health benefit is achieved at moderate intensity physical activity. However, this model gives us only a qualitative guideline. Specific recommendation cannot be derived. We will address this problem by introducing a quantitative model where benefit/risk profiles are parameterized, and activity intensity is measured by METs.

\section{Agent-Based Modeling}

As we will be using an agent-based model for studying the relationship between physical activity and health benefit/risk at both individual and societal level, we present a brief description of this approach here. Agent-based modeling, also known as individual based modeling, is a modeling approaches that represents system components and real-world entities via virtual agents. Agents may represent people, animals, resources, organizations, or any other entities. Agents can be heterogeneous, in which case, they have different properties and behavioral rules. Agent-based modeling is closely related to research in complex systems, emergence, computational sociology, multi-agent systems, evolutionary programming, and intelligent organizations (Bonabeau, 2002). The system's behavior emerges from the interaction of agents with other agents and the environment according to predefined behavioral rules (Wilensky and Rand, 2015).

Agent-based modeling is a natural way of modeling many real-world systems, such as biological organisms, ecosystems, organizations, markets, societies, political movements, and so on. It is an ideal model for studying individual choices. Researchers have used agent-based modeling to study how a macro phenomenon emerges from micro-level behaviors among a set of heterogeneous interacting agents. The ability to model individual styles and attributes, rather than assuming the homogeneity of the whole population, can be considered as one of the main advantages of Agent-based modeling over traditional mathematical equation-based approaches. Traditional models based on analytical techniques often become intractable when the complexity of systems approaches that of the real world. Furthermore, agent-based models can incorporate traditional models such as system dynamics, discrete event models, behavior models, etc., hence offering a flexible and powerful research tool for system analysis, scenario compassion, decision making, policy alternative studies, and public health intervention(North andMacal, 2007; Tracy et al., 2018).

\section{Individual Activity Model}

Nobody is the average person. Yet, most recommendations pushed by governments and health professionals are based on general population and the guidelines do not apply to individuals with their specific situations. Not only there are different health benefit profiles for different determinants of health (Warburton et al., 2016), but also the physical activity induced health benefit profiles are different from one individual to the next. This may be part of the reasons that most people do not meet the recommended guidelines. 
Simpson el al. (2003) reported that only $38.5 \%$ of US adults meet the national guidelines for physical activity. Current guidelines are too broad and generic that they cannot address the diverse needs of society. Warburton et al. (2016) recommended individualized exercise prescriptions for patients with varied recommendations for improving physical fitness and functional status. We believe that the same principle should be applied to healthy individual as well. That is, the physical activity recommendations should be built on top of the individual health benefit/risk profiles. Following the idea of Haskell (2001) as depicted in Figure 3, we establish the individual health benefit profile as a negative exponential function with two parameters $\alpha_{1}$ and $\beta_{1}$ that determine the curvilinear relationship between health benefit and the physical activity intensity measured in METs.

$$
\text { Benefit }=1-\alpha_{1} e^{-M E T / \beta_{1}}
$$

This benefit function supports the observation that health benefit has a steeper slope in the early stages of increased activity intensity. Additional benefits come from increasing activity intensity, but at a decreasing rate.Similarly, the individual risk profile can be established as an exponential function that depicts insignificant increase in risk as the activity intensity increases in the early stages. However, the risk level accelerates as the physical activity intensity approaches the maximum METs.

$$
\text { Risk }=\frac{1}{\alpha_{2}} e^{M E T / \beta_{2}}
$$

The parameters $\alpha_{1}, \alpha_{2}, \beta_{1}, \beta_{2}$ can be estimated from empirical data when the health benefit and risk are precisely defined, and data are collected. The feasibility of establishing the specific benefit/risk profiles is a subject of future research. In our study, we use simulated parameter values for distinctive individual profiles. Figure 4 shows an example of two health benefit profiles and one risk profile. BP1 (benefit profile 1) is linear. It is used as a base line to contrast BP2's benefit increase at a varied rate.

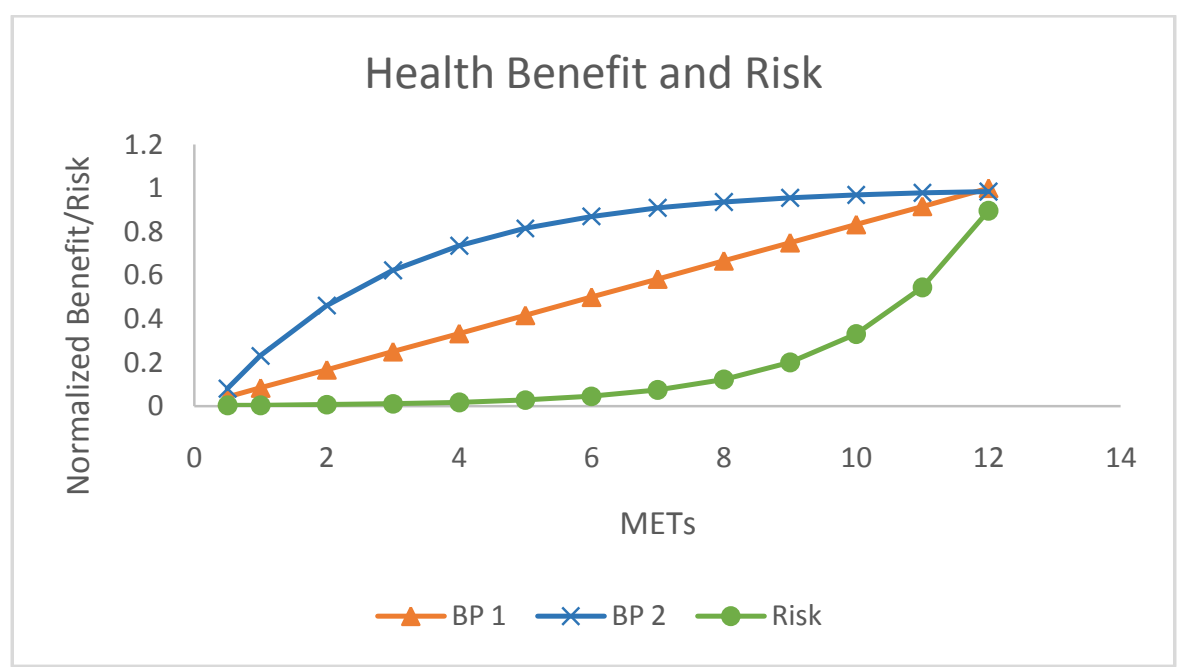

Figure 4. Typical Individual Health Benefit/Risk Profile

The net benefit for an individual is given by taking the difference between the health benefit and risk, as shown in the equation below:

$$
\text { Netbenefit }=1-\alpha_{1} e^{-M E T / \beta_{1}}-\frac{1}{\alpha_{2}} e^{M E T / \beta_{2}}
$$

Because different people have different health benefit and risk profiles, the maximum net benefit is achieved at different activity intensity level measured in METs. By taking derivative of the above net benefit function, it is straight forward to solve analytically for the METs value at which net benefit is maximized for each person.

$$
\text { METS }=-\frac{\beta_{1} \beta_{2} \ln \left(\frac{\beta_{1}}{\alpha_{1} \alpha_{2} \beta_{2}}\right)}{\beta_{1}+\beta_{2}}
$$

Keep in mind that METs computed above is for individuals, rather than for the population or certain groups of people, as we use individualized health benefit/risk profiles. Thus, each person is able to know his/her best physical activity intensity.Although some researchers believe that there are no lower or upper threshold for physical activity induced health benefit (Powell et al., 2011), there are evidences that prolonged strenuous physical activity are linked to various risks (Melzer et al., 2004; Warburton et al., 2016). 
Figure 5 shows a case where the risk profile leads to negative net benefit when the physical activity intensity is approaching the maximum.

\section{Health Benefit and Risk}

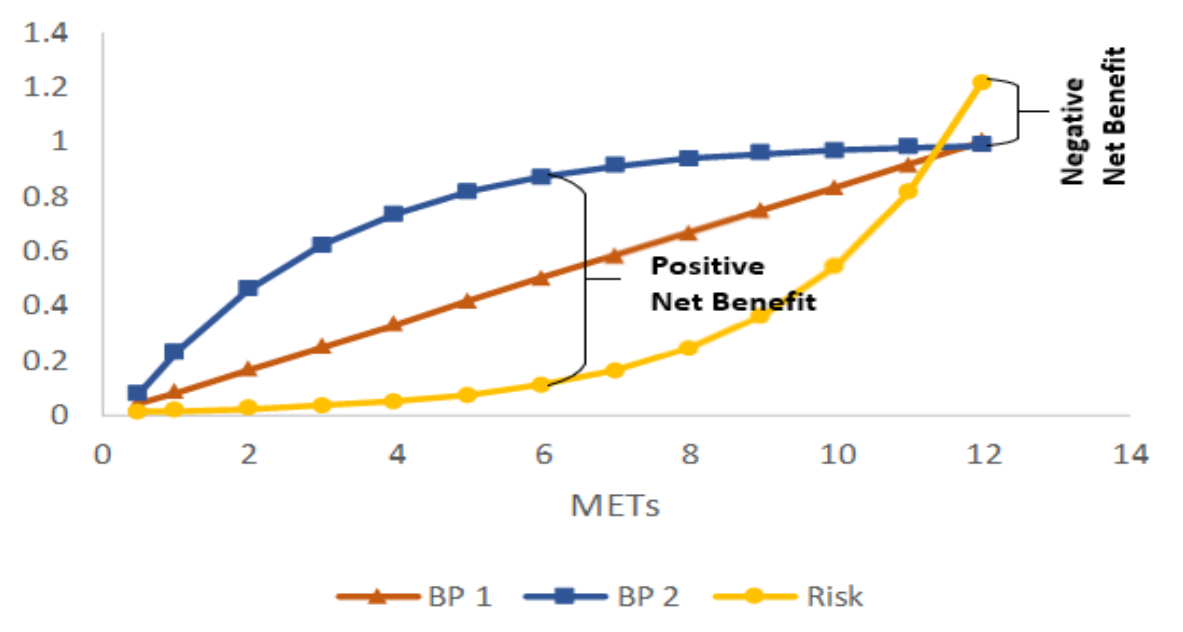

Figure 5. A Case Where Risk Exceeds the Benefit at 12 METs

\section{Model Simulation}

We implement the agent-based model in the NetLogo modeling and simulation platform. NetLogo is an open source software available from Northwestern University since 1999 (https://ccl.northwestern.edu/netlogo/). It was originally authored by Uri Wilensky and it has been maintained and developed by the Center for Connected Learning and Computer-Based Modelingat Northwestern University (Wilensky and Rand, 2015).

Toward the objective of reaching optimal health benefit, we assume that people start with a current physical activity level measured by METs with a uniform distribution ${ }^{1}$ around a national average METs. Based on their health benefit and risk profiles, they can establish a target METs value, as discussed in the previous section. In the simulation, the parameters are chosen as follows:

$\alpha_{1}=1.1$ ( \pm a random value in $[0,0.2]$

$\beta_{1}=2.8( \pm$ a random value in $[0,0.3]$

$\alpha_{2}=150( \pm$ a random value in $[0,50]$

$\beta_{2}=2.3$ ( \pm a random value in $[0,0.3]$

Since we assume that everyone is unique, and we use 1000 as the population size in the simulation, there are 1000 health benefit profiles and 1000 risk profiles. Figure 6 give a sample of the health benefit profiles and risk profiles used in the experiments.

A global level of METs can be set at any value in the range of 1 to 12. Initial METs for individual agents are set to be global METs plus/minus a value in [0, 2.0]. Individual METs are capped at 1 at the lower end and 12 at the upper end.

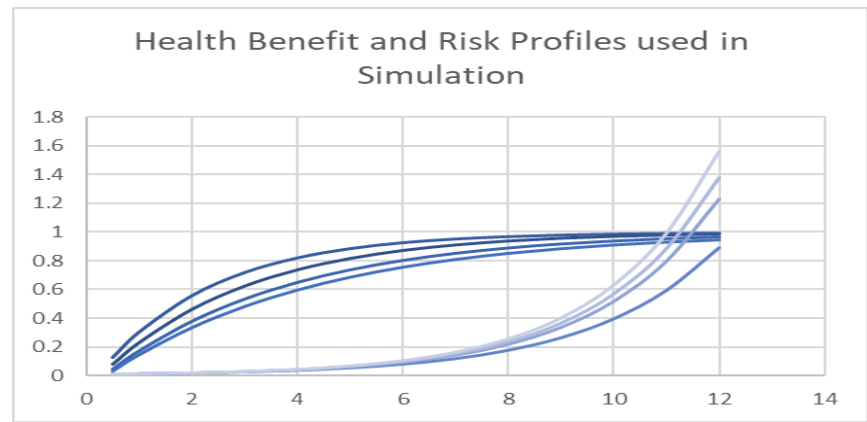

Figure 6. Sample Benefit and Risk Profiles

\footnotetext{
${ }^{1}$ Normal distribution is more appropriate. For simplicity, we use uniform distribution.
} 
As people adjust their physical activity intensity to gain better health benefits, it is reasonable to assume that the changes will be implemented in multiple stages. There will also be a lag in engaging physical activity and achieving desired health benefits. Thus, we introduce two control parameters in the simulation: 1) change factor, measured in percentage of the difference between current value and the target value; and 2) delay factor, measured in number of time units. These two parameters play an important role in the stability of system dynamics. A large change factor coupled with delay often lead to system instability.

\section{Simulation Results}

\subsection{Experiment 1: Population METs at 3, change factor at 30\%}

When the global METs is low, the initial risk is low, and the net benefit has a wide range of values. Figure 7 shows the initial distribution of health benefit, risk, net benefit, individual METs and their target METs.

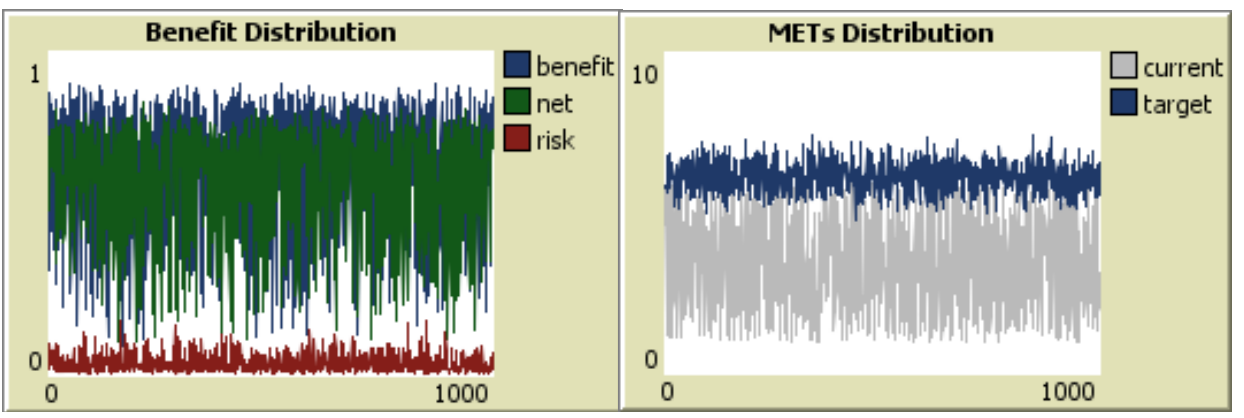

Figure 7. Initial Individual Value Distribution with Global METs = 3

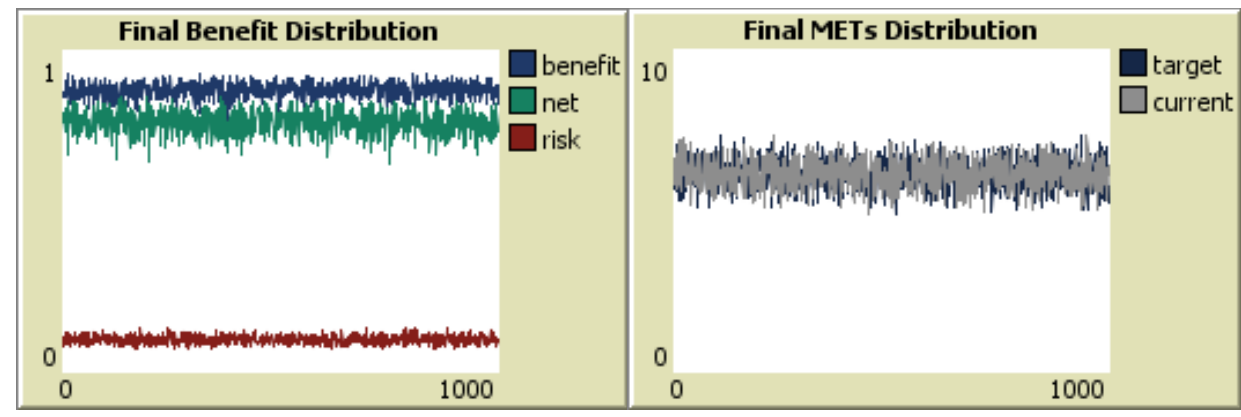

Figure8. Final Individual Value Distribution with Global METs = 3

When the delay is small, the system quickly converges to desired level of METs and achieves high societal level of net benefit, as shown in Figure 8. As the delay increases, it takes larger fluctuation and longer time for the system to stabilize. When delay moves from 4 to 5 units of time, the system become divergent. Figure 9 shows the progressive changes in population net benefits (average of individual net benefit) and average METs as delay increases from 0 to 5 .

The plots in Figure 9 present two population measures: the average net health benefit and the average METs. From the view point of policy making and national health promotion, those measures are of great interest. Other population health statistics of interest include the minimum and maximum values, the standard deviations, and the impact of certain national level interventions. For example, intervention programs could be developed to promote health of the vulnerable groups such as the elderly and the ill.

Figure 10 shows that there are similar patterns of convergence of health benefit for the average people, those in the upper echelon of health, and those in the lower echelon of health. However, the relative magnitude of improvement is different for people in different groups. Those in the lower echelon initially show significant increase in their health benefit. This is consistent with existing literature that inactive people or people with low level activity in general gain large health benefit when they engage in moderate physical activity (Powell, 2011; Warburton et al., 2016). Those people who are already active in physical activity have a relatively small amount of improvement, given their initial high level of heath. It would be interesting to see how reduced activity intensity would impact those already healthy individuals. This could be the subject of another research paper. 


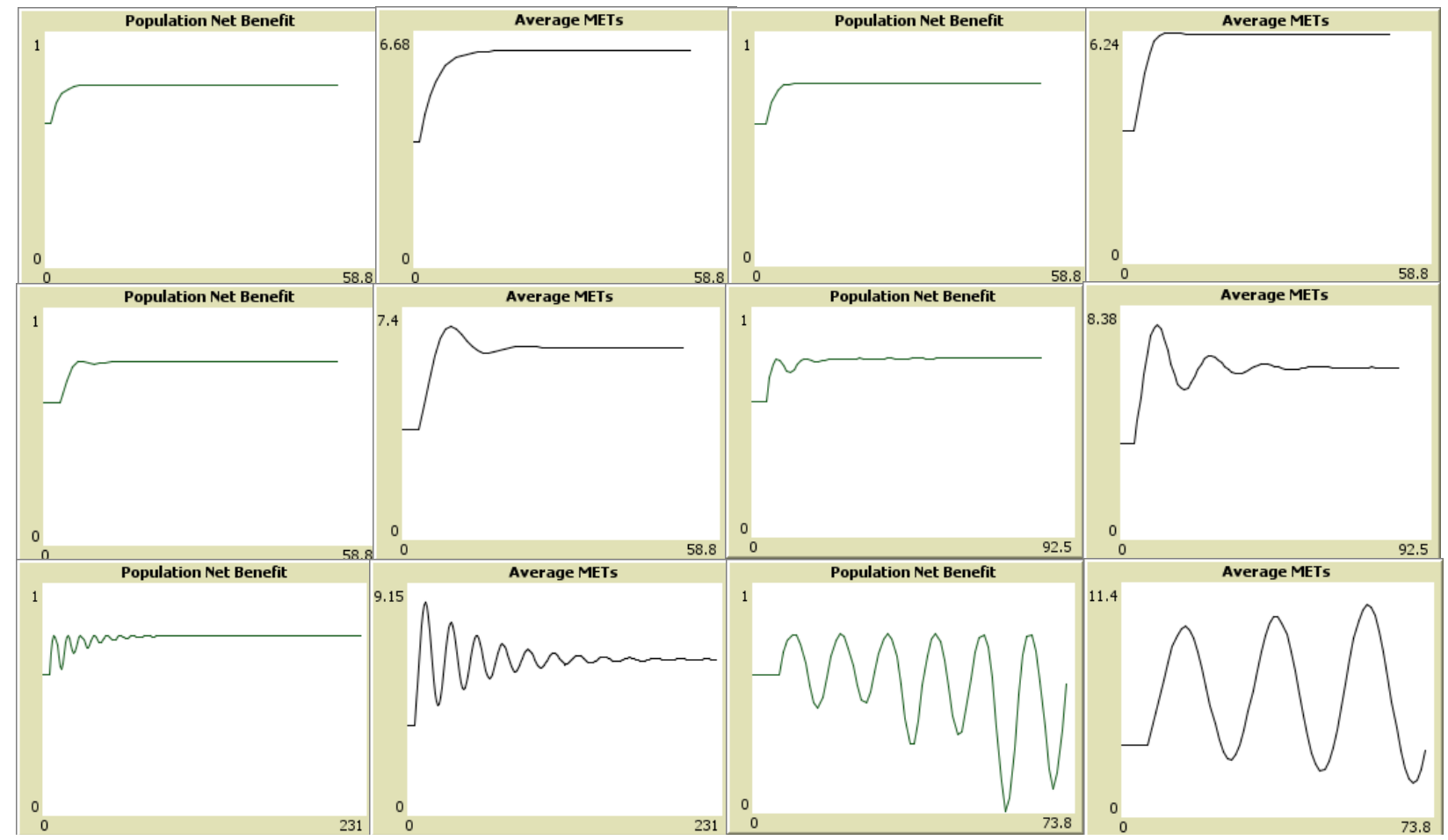

Figure 9. Population Benefits Move from Convergence to Divergence as Delay Increases (Top plots: delay 0 and 1; middle plots: delay 2 and 3; bottom plots: delay 4 and 5

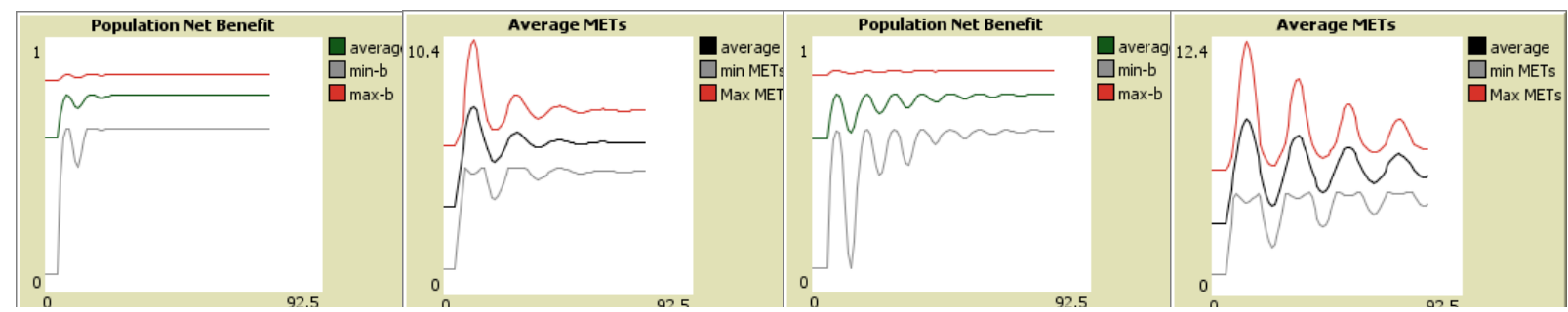

Figure 10. The Three Measures: Average, Minimum, and Maximum. Left, delay $=3$; right, delay $=4$

\subsection{Experiment 2: Population METs at 3, change factor at $15 \%$}

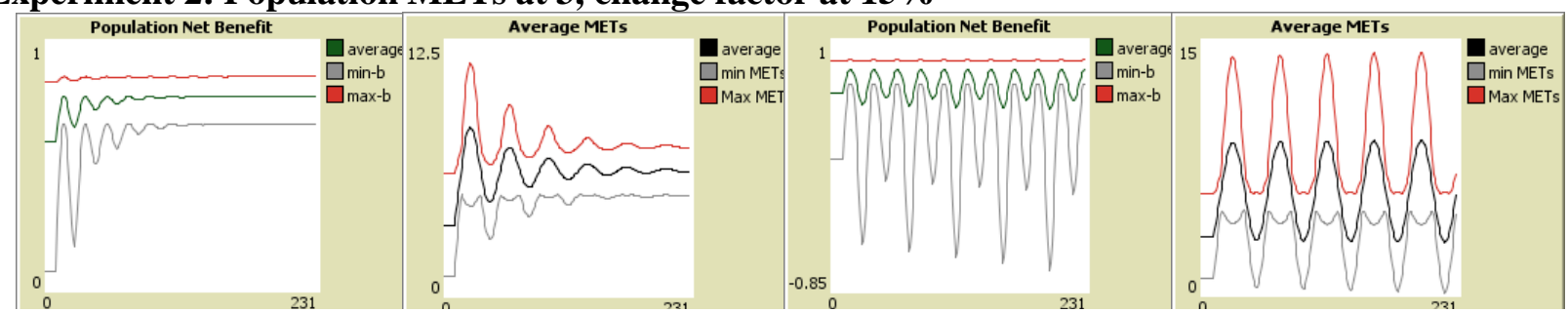

Figure 11. Smaller Change Factor with Longer Delay. Left, delay = 8; right, delay = 10

With a change factor of $30 \%$, the system become unstable when the delay reaches 5 units of time, as shown in Figure 9. To compensate for longer delay but still aim at reaching a stable system, we could reduce the change factor. Figure 11 shows that with a change factor at 15\%, the system still converges with a delay of 8 units of time. Instability is reached when the delay is at 10 units of time. Thus, smaller change factor helps to improve system stability when the gap between action and result is large.

\subsection{Experiment 3: Population METs at 6, change factor at $15 \%$}

METs lower than 3 is considered light physical activity. Clearly, as shown in Experiments 1 and 2, when the global METs level is low, there is significant room for improvement for most people. Nearly all people see increased activity level and improved net health benefit.

However, when the global METs is relatively high at the upper end of moderate physical activity $($ METs $=6)$, we see noticeable increase in risk level for some people. The desired level of METs based on health benefit profiles 
should be lower than the current METs for many people, as shown in Figure 12, because their initial METs are beyond the moderate level. Comparing Figure 12 to Figure 7, we see significant differences in the METs distribution and benefit/risk distribution.

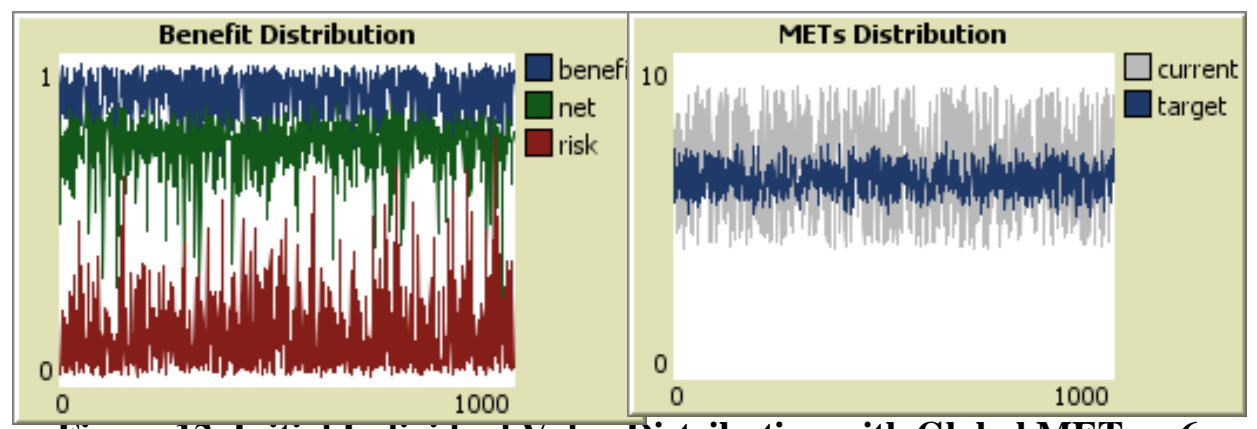

Figure 12. Initial Individual Value Distribution with Global METs = 6

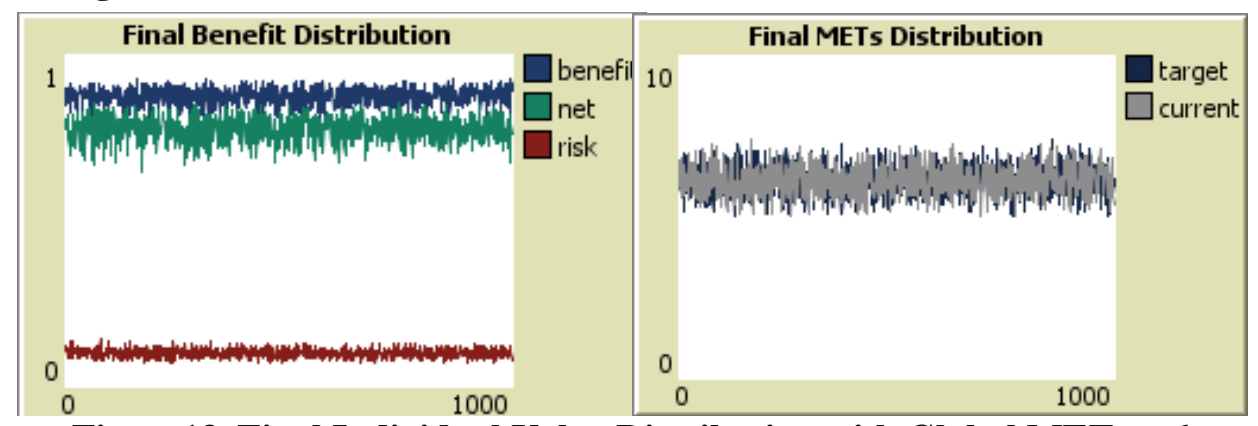

Figure13. Final Individual Value Distribution with Global METs $=6$

When the system converges, the final distributions of health benefit, risk, net benefit, current and desired METs are shown in Figure 13. Despite the significant differences in the initial distribution (Figure 7 vs Figure 12), the final distribution is nearly identical in Figure 13 and Figure 8. This is the desired effect: when the system converges, the optimal health level is achieved while initial parameters are different (METs 3 vs. 6; change factor $30 \%$ vs. 15\%). The implication is that initial global METs is not important to the final societal level of net health benefit, given that the system converges to a stable equilibrium. But the change factor and delay are significant in determine the system's stability as shown in Figure 9 and Figure 11.

When the delay is less than 10 units of time, the system converges to stable level of METs and net health benefit. Figure 14 shows the system dynamics with the delay set at 8 and 10 units. The same as the case when the global METs $=3$, the system becomes unstable when the delay reaches 10 units or above. But there are noticeable differences in the change dynamics.

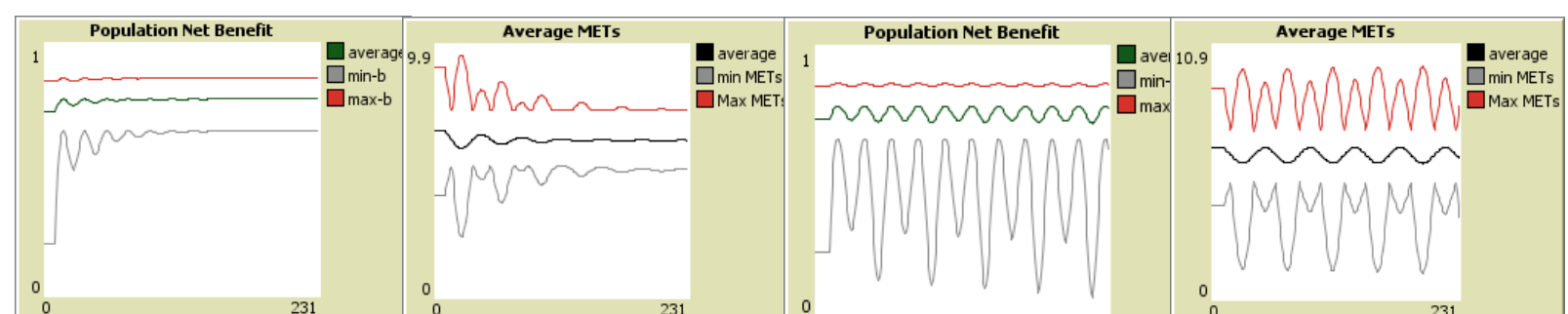

Figure 14. Smaller Change Factor with Longer Delay. Left, delay = 8; right, delay = 10

\section{Discussion}

The World Health Organization has estimated that physical inactivity is the fourth leading risk factor for global mortality (WHO, 2010). Governments and health professional organizations have encouraged physical activity by issuing guidelines for the general populations. Recognizing the limitations of current broad guidelines and recommendations (Warburton et al., 2016), we propose to use individualized health benefit/risk profiles to study the desirable level of physical activity at both personal level and societal level. Our preliminary simulation results indicate that the agent-based model provides valuable insights regarding the population health benefit/risk distribution, the population net benefit change dynamics, the system stability as influenced by the magnitude of changes and the delayed realization of target result through the implementation of changes. 
Agent-based modeling has been deployed in various research for several decades. Tracy et al. (2018) presented a review of current applications and future directions of agent-based modeling inpublic health and concluded that despite some limitations and challenges, agent-based modelingoffers a promising tool for informing public health research, practice, and policy. Our research is an effort in bring a new perspective in studying the relationship between physical activity and health benefit/risk. However, this research is limited in scope and depth. There are many unexplored research questions.

\section{Contributions}

The main contributions of this research are: 1) Turning previous qualitative relationship between physical activity and health benefit/risk into quantitative health benefit/risk profiles. 2) Developing an agent-based model for study societal health benefit with heterogeneous population. 3) Incorporating system dynamics in societal health benefit stability.

\section{Limitations}

There are several limitations of the current research due to its preliminary nature. 1) The quantitative model, although performing reasonably well in the simulations, lacks empirical validation. Finding the exact benefit/risk profile for everyone effectively and efficiently may not be practical. 2) Using METs to measure physical activity does not consider the impact of activity time. A few minutes of vigorous activity (METs > 6) is not as good as one hour of moderate activity $(3 \leq$ METs $\leq 6)$. 3) The current study does not consider the dynamic nature of individual optimal activity level. For simplicity, we use fixed parameters for individual health benefit/risk profiles. But in the real world, the benefit/risk profile for each person is different at different stage of his/her life. 4) Human behavior is influenced by both internal traits (such as personality) and external influences (such as mass media and peer pressure). The current model has not implemented those significant factors.

Despite the limitations, we believe our proposed model has untapped potentials in contributing to the study of the relationship between physical activity and health benefit/risk, short-term and long-term national guidelines/recommendation, health intervention policies. For example, even though the present study uses only fixed form of benefit profiles and risk profiles, agent-based modeling can easily incorporate other types of profiles such as sigmoidal, polynomial, step-function, etc.Different or mixed functional forms of health benefit/risk profiles might be applicable for different groups of people, separated by age, gender, heritage, culture, or socialeconomic status. Scenarios analysis can be performed to evaluate policies or intervention programs that directly or indirectly impact the health benefit/risk profiles. Accessibility to sports facilities or other resources that are conducive to physical activity can be integrated with the agent-based model.

Further research will be carried out to test systematically the impact of control factors on the societal health benefit and system stability. Other future research topics will be aimed at addressing some of the limitations of the current model.

\section{Conclusion}

Good health at both the individual level and at society level is of vital importance. It has long been established that regular moderate physical activity is a crucial contributor to robust physical health. However, most conventional physical activity guidelines and recommendations are designed to address the broad population or groups of people. The relevance and effectiveness of those recommendations may not be appropriate when applied to individuals with substantially different activity induced health profiles. We recommend that tailored recommendations be built upon individual health profiles to achieve maximum benefit.

Our preliminary research on using agent-based modeling for studying the relationship between physical activity and health benefit/risk has provided new insights in integrating individual physical activity choices with societal level health benefit. Further enhancement and validation of this model are needed. However, the initial work shows that this approach offers power, flexibility, and potentials in helping us to gain better understandings of physical activity and public health. 


\section{References}

Ainsworth BE, Haskell WL, Leon AS, Jacobs DR Jr, Montoye HJ, Sallis JF, Paffenbarger RS Jr. (1993). Compendium of physical activities: classification of energy costs of human physical activities, Medicine and Science in Sports and Exercise, 25(1), 71-80.

BonabeauE. (2002). Agent-based modeling: Methods and techniques for simulating human systems. Proc. National Academy of Sciences, 99, 7280-7287.

Conservapedia. Available:https://www.conservapedia.com/Plato. Accessed May 18, 2018.

Gledhill, N. andJamnik, V. (2003). Canadian Physical Activity, Fitness and Lifestyle Approach, 3rd ed. Canadian Society for Exercise Physiology: Ottawa, Canada.

Haskell, W. L. (2001). What to look for in assessing responsiveness to exercise in a health context. Medicine Science in Sports and Exercise. Medicine Science in Sports and Exercise, 33(Suppl), S454-458.

Haskell, W. L., Lee, I-M., Pate, R. R., Powell, K. E., Blair, S. N., Franklin, B. A., ... Bauman, A. (2007). Physical activity and public health: Updated recommendation for adults from the American College of Sports Medicine and the American Heart Association. Medicine and Science in Sports and Exercise, 39(8), 1423-1434.

Kesaniemi YK, Danforth E Jr, Jensen MD, Kopelman PG, Lefèbvre P, Reeder BA. (2001). Dose-response issues concerning physical activity and health: an evidence-based symposium, Medicine Science in Sports and Exercise, 33(6 Suppl), S351-358

Kujala UM.(2009). Evidence on the effects of exercise therapy in the treatment of chronic disease British Journal of Sports Medicine,43, 550-555.

Melzer, K., Kayer, B., and Claude, P. (2004). Physical activity: the health benefits outweigh the risks, Current Opinion in Clinical Nutrition and Metabolic Care, 7, 641-647.

North, M. J., \&Macal, C. M. (2007). Managing business complexity: Discovering strategic solutions with agentbased modeling and simulation. New York: Oxford University Press.

O'Donovan, G, Blazevich AJ, Boreham C., Cooper AR., Crank H., Ekelund U., Fox KR., Gately P., Giles-Corti B., Gill JMR., Hamer M., McDermott I., Murphy M., Mutrie N., Reilly JJ., Saxton, JM.\&Stamatakis E. (2010). The ABC of Physical Activity for Health: A consensus statement from the British Association of Sport and Exercise Sciences, Journal of Sports Sciences, 28:6, 573-591.

Powell, KE, Paluch, AE, and Blair SN. (2011). Physical Activity for Health: What Kind? How Much? How Intense? On Top of What? Annual Review of Public Health, 32,349-365.

Simpson ME, Serdula M, Galuska DA, Gillespie C, Donehoo R, Macera C, Mack K.(2003).Walking trends among U.S. adults: The Behavioral Risk Factor Surveillance System, 1987-2000. American Journal of Preventive Medicine, 25(2), 95-100.

Tracy, M., CerdM., and Keyes, KM. (2018). Agent-Based Modeling in Public Health: Current Applications and Future Directions. Annual Review of Public Health, 39, 77-94.

U.S. DoHHS.(2008). Physical Activity Guidelines for Americans. U.S. Department of Health and Human Services, ODPHP Publ. No. U0036. Available: http://www.health.gov/paguidelines/pdf/paguide.pdf. Accessed May 18, 2018.

Wilensky U. and Rand W. (2015). An Introduction to Agent-Based Modeling: Modeling Natural, Social, and Engineered Complex Systems with NetLogo, The MIT Press, Cambridge, MA

Warburton, D.E.E., Taunton, J., Bredin, S.D., Isserrow, S.H. (2016). The risk-benefit paradox of exercise BE Medical Journal, 58, 210-218.

WHO. (2010). Global recommendations on physical activity for health. Geneva: World Health Organization. Available: http://apps.who.int/iris/bitstream/handle/10665/44399/9789241599979_eng.pdf. Accessed May 18, 2018. 